\title{
FIT: the scintillating fiber tracker of the HERD space mission
}

\section{Perrina, ${ }^{a, *}$ P. Azzarello, ${ }^{b}$ F. Cadoux,${ }^{b}$ Y. Favre, ${ }^{b}$ J. M. Frieden, ${ }^{a}$ D. La Marra, ${ }^{b}$ D. Sukhonos ${ }^{b}$ and $\mathrm{X}$. Wu $\mathbf{W}^{b}$ on behalf of the HERD Collaboration (a complete list of authors can be found at the end of the proceedings)}

${ }^{a}$ Institute of Physics, Ecole Polytechnique Fédérale de Lausanne (EPFL), CH-1015 Lausanne, Switzerland ${ }^{b}$ Département de Physique Nucléaire et Corpusculaire (DPNC), Université de Genève, CH-1211 Genève 4, Switzerland

E-mail: Chiara.Perrina@epfl.ch

The High Energy cosmic-Radiation Detection (HERD) facility is a space payload proposed to be installed onboard the China's Space Station (CSS). The aims of HERD are the indirect detection of dark matter, the direct detection of cosmic rays towards the "knee" of the spectrum $(\sim 1 \mathrm{PeV})$ and the monitoring of the full gamma-ray sky from $100 \mathrm{MeV}$. The HERD core is a calorimeter capable of accepting particles incident on its top and four lateral sides, each equipped with a sector of the scintillating fiber tracker: FIT. The FIT sectors host 7 tracking planes made of modules. The module, composed of a fiber mat and three arrays of silicon photomultipliers (SiPMs), is the elementary brick of FIT. Several FIT modules have been built and tested with particle beams at CERN. A FIT demonstrator, made of two partially instrumented tracking planes, has been assembled and sent through vibration tests. The results of the performed tests as well as the current design of FIT are presented in this contribution.

$37^{\text {th }}$ International Cosmic Ray Conference (ICRC 2021)

July 12th - 23rd, 2021

Online - Berlin, Germany

\footnotetext{
${ }^{*}$ Presenter
} 


\section{Introduction}

The High Energy cosmic-Radiation Detection (HERD) facility is a particle space borne detector, planned to be operational starting from 2027 for about 10 years onboard the China's Space Station (CSS). The objectives of HERD are: the search for annihilation and decay products of dark matter, both in the energy spectrum and anisotropy of electrons and positrons from $10 \mathrm{GeV}$ to $100 \mathrm{TeV}$ and in the gamma-ray spectrum; the measurement of the energy spectrum and composition of cosmic rays (protons and heavier nuclei up to iron) from $30 \mathrm{GeV}$ to a few $\mathrm{PeV}$; a wide field of view monitoring of the $\gamma$-ray sky from $100 \mathrm{MeV}$. HERD is composed of five subdetectors (Fig. 1): the innermost instrument is a deep (55 radiation lengths and 3 nuclear interaction lengths) 3D imaging calorimeter (CALO), made of about 7500 LYSO cubes with an edge length of $3 \mathrm{~cm}$, arranged into an octagonal prism, which measures the energy of the particles entering the detector from five sides except the bottom, distinguishing electrons from hadrons. The top side and the four lateral sides of the calorimeter are instrumented from inside out with a sector of the following detectors:

- the scintillating FIber Tracker (FIT), which is described in detail in the next section;

- the Plastic Scintillator Detector (PSD), which measures the absolute value of the charge $(|Z|)$ of cosmic rays and acts as an anti-coincidence system for charged particles in the gamma-ray detection;

- the Silicon Charge Detector (SCD), which provides an independent measurement of the absolute value of the charge $(|Z|)$ before any charge-change interaction could occur.

On one lateral sector of the SCD is located the Transition Radiation Detector (TRD) needed to calibrate the response of the calorimeter to high energy hadronic showers.

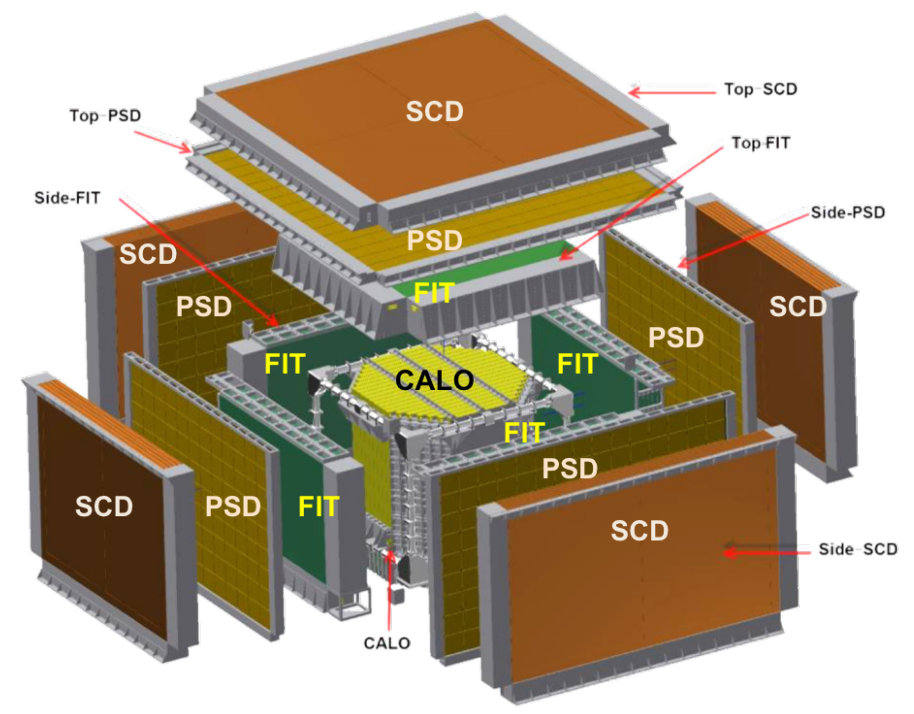

Figure 1: Exploded view of the HERD detector. The innermost subdetector is the calorimeter (CALO), surrounded on the top and the four lateral sides by the scintillating FIber Tracker (FIT), the Plastic Scintillator Detector (PSD) and the Silicon Charge Detector (SCD). 


\section{The scintillating FIber Tracker (FIT)}

The HERD scintillating FIber Tracker (FIT) is being designed for the purpose of reconstructing the trajectories and the charge absolute value $(|Z|)$ of charged cosmic rays, favour the conversion of low energy gamma rays and reconstruct the tracks of the generated electrons and positrons. Simulation studies are ongoing aiming at designing the tracker with the best performance for geometrical acceptance, angular resolution and converting capability while keeping the structure mechanically stable and meeting the thermal and power conditions requested by the space station. In the current design, FIT (Fig. 2a) consists of 5 tracking sectors (Fig. 2b), made of 7 tracking planes (Fig. 2c), for 7 independent measurements of the position of a traversing charged particle. Each tracking plane consists of two layers of FIT modules measuring the two orthogonal spatial coordinates. The tracking planes of the top sector are made of 10 FIT modules on both the $\mathrm{x}$ layers and $y$ layers, while the tracking planes of the side sectors are made of 6 FIT modules on the $\mathrm{x}$ layers and 10 FIT modules on the y layers. A module (Fig. 2d) includes one scintillating fiber mat and three silicon photomultiplier (SiPM) arrays to read out the scintillation light induced by the particles hitting the mat. The mat is made by stacking 6 layers of fibers for a width of $97.8 \mathrm{~mm}$ to match three S13552-10 SiPM arrays. The latter is a customized array of $128 \mathrm{SiPMs}$ produced by Hamamatsu Photonics ${ }^{1}$. Each channel contains $3749(23 \times 163)$ pixels of $10 \mu \mathrm{m} \times 10 \mu \mathrm{m}$ area. Each SiPM array is mounted on a small printed circuit board (PCB) screwed to the end-pieces of the fiber mat (Fig. 2e). The three PCBs are connected to the main body of the front-end board (Fig. 2f) through flexible cables. On the back side of the SiPM PCB is mounted a PT100 thermal sensor, to allow for the correction of the bias voltage according to the temperature. In this configuration, FIT has 225792 read-out channels. So far the 128 channels of each SiPM array are read out by two VATA64 HDR16.2 ASIC chips made by IDEAS ${ }^{2}$ that have a power consumption of about $9 \mathrm{~mW} / \mathrm{channel}$. A dedicated ASIC with larger dynamic range and a power consumption less than $1 \mathrm{~mW} /$ channel is being developed by the Institute of Cosmos Sciences of the University of Barcelona. This ASIC will have a dynamic range sufficient for charge measurements up to $Z=26$. More details about the design of the front-end board and the FIT Read-out Board (FRB) can be found in [1]. The 7 tracking layers of each sector are mounted on 7 support trays. A support tray consists of a $20 \mathrm{~mm}$ thick core of aluminum honeycomb sandwiched between two $0.6 \mathrm{~mm}$ thick sheets of Carbon Fiber Reinforced Polymer (CFRP).

\section{The FIT module}

The active area of a FIT module is the fiber mat which is composed of six layers of plastic scintillating fibers arranged tightly in parallel along the length. The fibers, manufactured by Kuraray $^{3}$ (type SCSF-78MJ), with a polystyrene core surrounded by two claddings, have a round section with an average total diameter of $250 \mu \mathrm{m}$. Such a fiber mat has the same characteristics of the fiber mats designed and used for the LHCb tracker upgrade [2], except that the width and length were both reduced to meet the detector dimension requirements. The fiber mat is read out by three

\footnotetext{
${ }^{1}$ https://www.hamamatsu.com

${ }^{2}$ https://ideas.no

${ }^{3}$ https://www.kuraray.com
} 


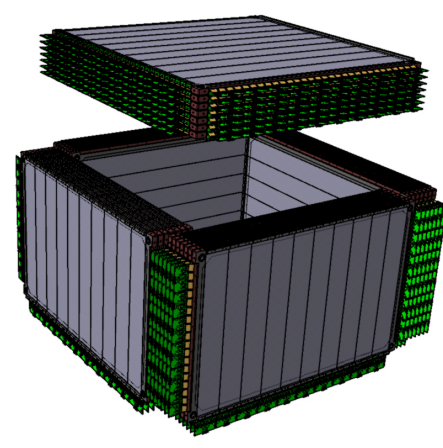

(a)

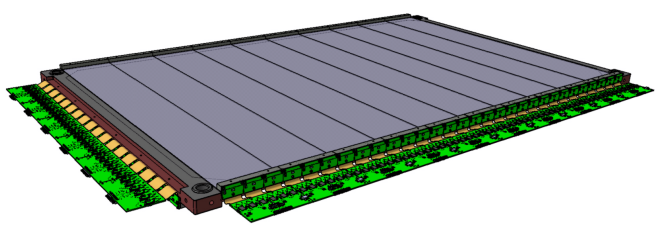

(c)

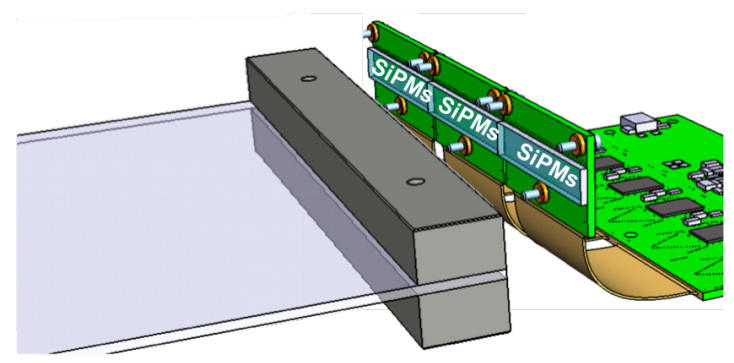

(e)

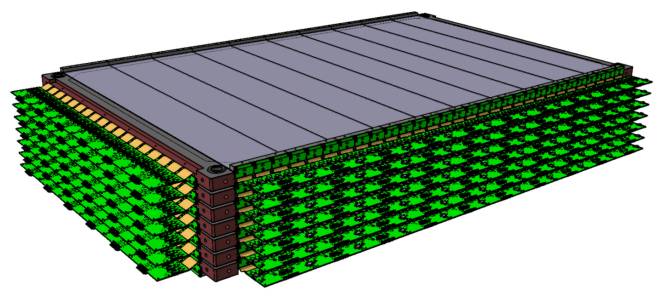

(b)

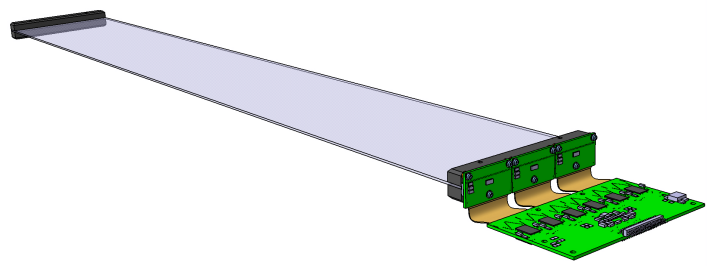

(d)

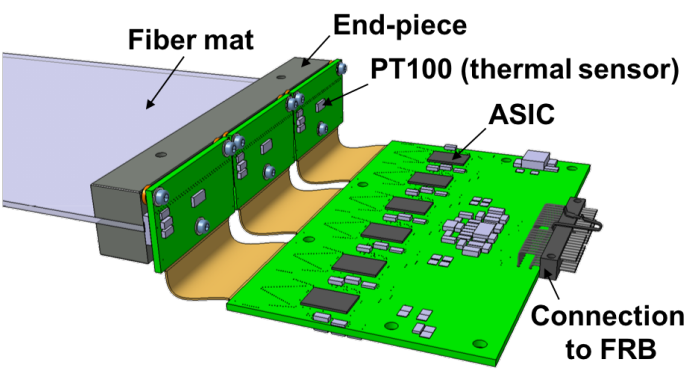

(f)

Figure 2: (a) Sketch of the 5-side FIT detector made of 4 lateral sectors and a top sector. (b) A lateral sector with 7 tracking planes. (c) A lateral tracking plane made of $6 \times 10$ FIT modules. (d) A FIT module made of a scintillating fiber mat and three SiPM arrays mounted on the front-end board. (e) and (f) Details of the front-end board coupled to the fiber mat.

"S13552-10" SiPM arrays. They are based on the "S13552-HRQ" developed for the LHCb tracker upgrade [3] and contain $128 \mathrm{SiPMs}$ each. While in the LHCb SiPMs there are 104 pixels, in the HERD SiPMs there are 3749 pixels $(23 \times 163)$ of $10 \mu \mathrm{m} \times 10 \mu \mathrm{m}$ area. The larger number of pixels is required by HERD since FIT should also measure the particle absolute charge in addition to the reconstruction of the trajectory. Taking into account the $20 \mu \mathrm{m}$ gap between neighbor SiPMs, the horizontal pitch of each channel is $250 \mu \mathrm{m}$, equivalent to the fiber diameter but slightly smaller than the fiber pitch. A epoxy layer with $100 \mu \mathrm{m}$ thickness is deposited on the top of the SiPM surface. Fig. 3 shows the photograph of a FIT module, where the fiber mat is covered with a Kapton black tape. 


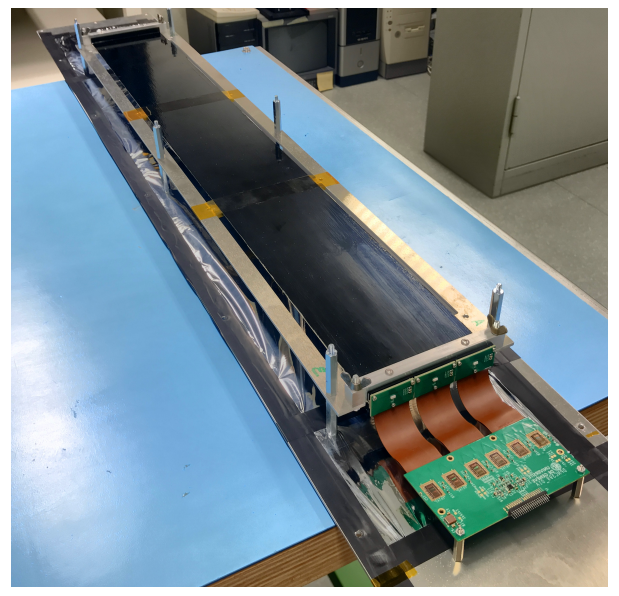

Figure 3: Photograph of a FIT module.

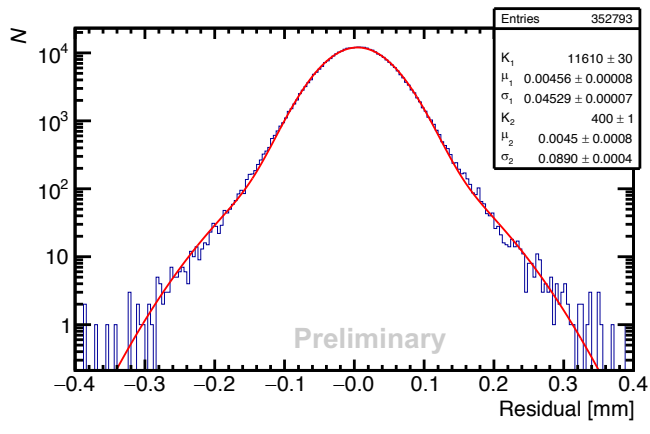

(a)

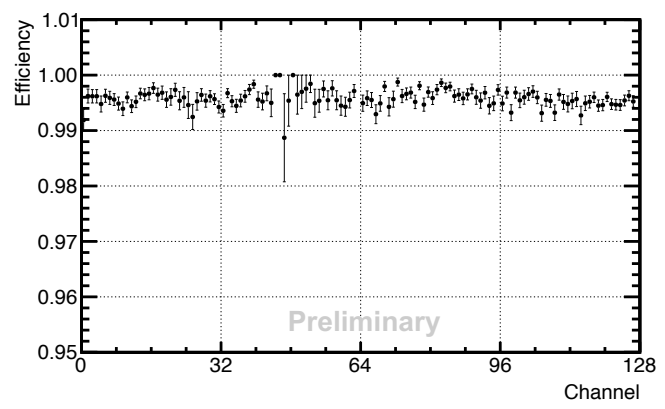

(b)

Figure 4: (a) Residual distribution for the reconstructed tracks. The red curve, representing the sum of two Gaussian functions, is the best fit to the data. (b) Hit detection efficiency of the 128 SiPMs of the array.

A FIT module prototype made of a $77 \mathrm{~cm}$ long fiber mat and one SiPM array was exposed to a $400 \mathrm{GeV} / \mathrm{c}$ primary proton beam and a fragmentation ion beam created by a $150 \mathrm{AGeV} / \mathrm{c}$ lead beam hitting a beryllium target at CERN. A spatial resolution of $45 \mu \mathrm{m}$ (Fig. 4a) and a mean hit efficiency of $99.6 \%$ (Fig. 4b) were measured for protons. The response to nuclei from helium up to beryllium was studied and a charge resolution better than $15 \%$ was found.

In order to evaluate the performance of a FIT sector in charge measurements, a multi-plane simulation was implemented ([4]). A correction algorithm was introduced to homogenize the signal of isotropically incident particles. Afterwards, the corrected charge resolution of the FIT sector with different number of tracking planes was calculated. Fig. 5 shows the charge resolution of the different configurations as a function of the nuclei charge $(Z)$. The charge resolution improves by increasing the number of tracking planes. The combined charge resolution of $5 \mathrm{x}$-y tracking planes is 0.11 charge units (c.u.) for carbon, 0.19 c.u. for silicon, and 0.32 c.u. for iron. For reference, the charge measurement performance of an AMS-02 single layer (double-sided measurement) and the AMS-02 inner tracker (L2-L8) [5] is also plotted in Fig. 5. The comparison shows that the performance of the 9 FIT planes is better than the AMS-02 inner tracker, which is a combination of 
7 double-sided silicon micro-strip layers.

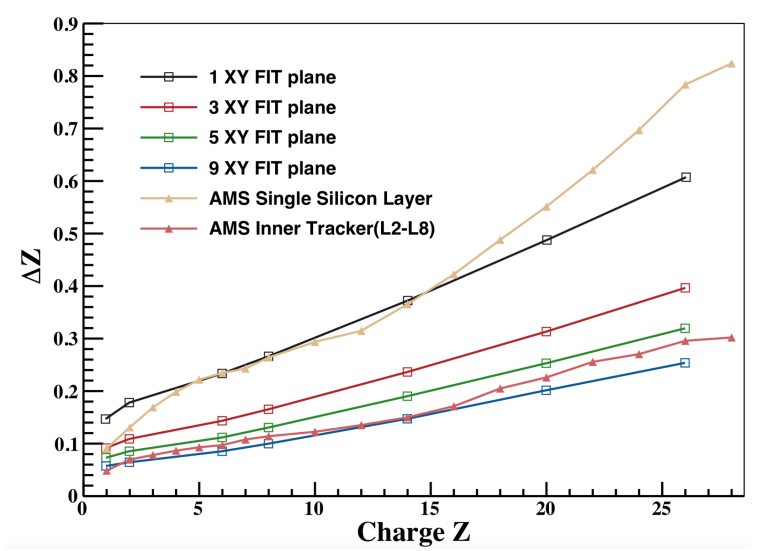

Figure 5: Simulated charge resolution of the FIT sector with different number of tracking planes as a function of the nuclei charge $\mathrm{Z}$. The charge resolution for one AMS silicon layer and for the AMS inner tracker is also shown for comparison.

\section{The FIT demonstrator and its space qualification}

A demonstrator composed of three trays partially equipped with fully working FIT modules was assembled. Four front-end electronics boards based on the VATA64 HDR16.2 ASIC, as well as two FRB boards have been produced. The demonstrator is being submitted to space qualification tests (vibration, thermal cycling, thermal vacuum tests). The tests are in progress and should be completed by the end of 2021. The tray design concept is based on the DAMPE STK tray [6]. The trays are made of an aluminum honeycomb structure sandwiched between two CFRP face sheets. The tray frames and the corner elements are made of CFRP bars. The tray design and production has been done in collaboration with Composite Design ${ }^{4}$. In total three trays have been produced. Two are made with a skin of $0.6 \mathrm{~mm}$, while the third one has a skin of $0.3 \mathrm{~mm}$, to test a design with a lighter weight. Before mounting the fiber mats, the trays have been thermally cycled in a range from $-30{ }^{\circ} \mathrm{C}$ to $60{ }^{\circ} \mathrm{C}$. To check possible structural changes, after each thermal test the tray eigenfrequencies have been measured with a smart hammer test system. For each test, an accelerometer was placed in a corner and at the center of the tray. The measured spectral power density distribution allowed to identify the tray eigenfrequencies which did not significantly change during the various steps of the test, thus confirming the stability of the tray structure. The fit module production has been done in two steps. First, 18 modules made of fishing lines have been made, to test the production procedures and the assembly tools. Then 16 modules made of scintillating fibers have been produced. The FIT modules have been sequentially glued on the trays. Glue dots have been deposited through a mask on each FIT module, which was then placed on the tray. The module positioning on the tray has been done using an alignment pin inserted into the module end piece guiding the FIT module thanks to a corresponding hole on the tray.

${ }^{4}$ https://www.compositedesign.ch 


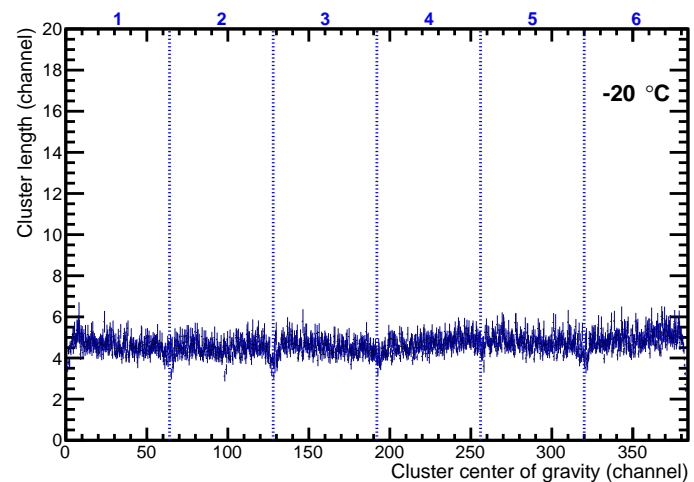

(a)

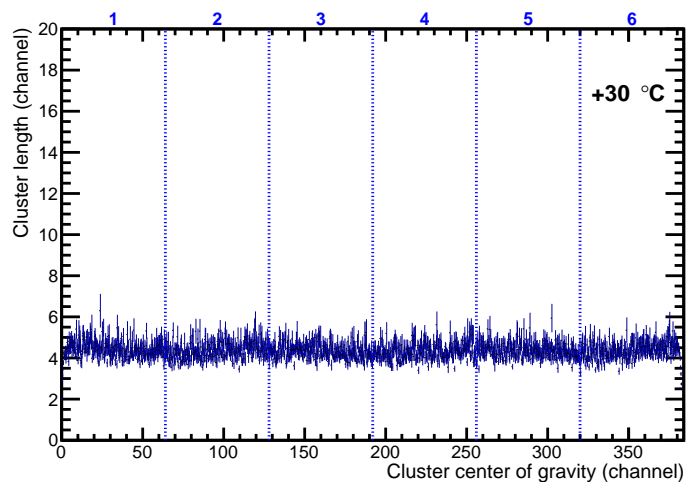

(b)

Figure 6: Cluster length as a function of the cluster center of gravity for atmospheric particles at $-20{ }^{\circ} \mathrm{C}($ a) and at $30{ }^{\circ} \mathrm{C}(\mathrm{b})$.

\subsection{Thermal vacuum tests of FIT modules}

A custom thermal vacuum setup has been developed to test FIT modules: a $1.5 \mathrm{~m}$ long vacuum cylinder has been installed in a thermal test chamber. In good testing conditions the vacuum can reach values as low as $5 \times 10^{-6}$ mbar, even if the vacuum level also depends on the outgassing of the tested material. Scintillators have been installed on the top and bottom of the thermal chamber, so that the detectors under vacuum can be tested with cosmic rays. Different run acquisitions were taken from $-20{ }^{\circ} \mathrm{C}$ to $30{ }^{\circ} \mathrm{C}$ with steps of $10{ }^{\circ} \mathrm{C}$. A set of 12 PT100 temperature sensors was also available, allowing to monitor the chamber and detector temperature at different positions. Figs. $6 \mathrm{a}$ and $6 \mathrm{~b}$ show that the cluster length for cosmic events registered at the two limit temperatures has not changed thus indicating a good mechanical stability at the interface between the SiPM arrays and the fiber mat.

\subsection{Vibration tests of the FIT demonstrator}

A first vibration test of the FIT demonstrator has been successfully completed at the Space Research and Planetology Division of the University of Bern, showing no significant change in the FIT demonstrator structure. The scintillating fiber modules have also been tested and show no change in the performance. Finally, one front-end electronics board has also been vibrated, and has remained fully functional after the test.

\section{Conclusions}

FIT is the scintillating fiber tracker of the HERD space mission. It serves for the track reconstruction of the charged cosmic particles, for the conversion of low energy gamma rays and for a redundant measurement of the particle charge $(|Z|)$. To study the tracking performance, the detector was exposed to the SPS $400 \mathrm{GeV} / \mathrm{c}$ primary proton beam at CERN. The detector spatial resolution was estimated at $45 \mu \mathrm{m}$ while the mean single hit detection efficiency is $99.6 \%$. The charge identification capability was evaluated during a second test campaign at CERN, using a fragmentation ion beam. The analysis indicates that up to beryllium, i.e. $Z<5$, the charge 
resolution is better than $15 \%$ (the measurement is limited to $Z<5$ due to the test read-out electronics). The space qualification process is ongoing and the first tests provide a confirmation of the mechanical stability of the different FIT elements.

\section{Acknowledgments}

The authors gratefully acknowledge the financial support from the Swiss Space Office of the State Secretariat for Education, Research and Innovation (ESA PRODEX Programme) and the Swiss National Science Foundation (SNSF).

\section{References}

[1] C. Perrina et al., The Tracking System of HERD, in Proceedings of 36th International Cosmic Ray Conference - PoS(ICRC2019), vol. 358, p. 122, 2019, https://pos.sissa.it/358/122/.

[2] C. Joram, U. Uwer, B.D. Leverington, T. Kirn, S. Bachmann, R.J. Ekelhof et al., LHCb Scintillating Fibre Tracker Engineering Design Review Report: Fibres, Mats and Modules, Tech. Rep. LHCb-PUB-2015-008. CERN-LHCb-PUB-2015-008, CERN, Geneva (Mar, 2015).

[3] O. Girard, G. Haefeli, A. Kuonen and M.E. Stramaglia, Silicon photomultiplier multichannel arrays for the LHCb scintillating fibre tracker, in 2017 IEEE Nuclear Science Symposium and Medical Imaging Conference, 2017, DOI.

[4] J.-j. Wang, X. Wu, M. Xu, C. Perrina, P. Azzarello, F. Cadoux et al., Feasibility study of cosmic-ray components measurement by using a scintillating fiber tracker in space, Radiation Detection Technology and Methods (2021) .

[5] Y. Jia, Q. Yan, V. Choutko, H. Liu and A. Oliva, Nuclei charge measurement by the Alpha Magnetic Spectrometer silicon tracker, Nuclear Instruments and Methods in Physics Research A 972 (2020) 164169.

[6] P. Azzarello et al., The DAMPE silicon-tungsten tracker, Nuclear Instruments and Methods in Physics Research A 831 (2016) 378. 


\section{Full Authors List: HERD Collaboration}

O. Adriani ${ }^{26}$, F. Alemanno ${ }^{27}$, R. Aloisio ${ }^{27}$, C. Altomare ${ }^{23}$, G. Ambrosi ${ }^{34}$, Q. An ${ }^{10}$, M. Antonelli ${ }^{45}$, P. Azzarello ${ }^{37}$, L. Bai ${ }^{8}$, Y.L. Bai ${ }^{3}$, T.W. Bao ${ }^{1}$, M. Barbanera ${ }^{34}$, F.C.T. Barbato ${ }^{27}$, P. Bernardini ${ }^{30}$, E. Berti ${ }^{26}$, B. Bertucci ${ }^{35}$, X.J. Bi ${ }^{1}$, G. Bigongiari ${ }^{36}$, M. Bongi ${ }^{26}$, V. Bonvicini ${ }^{45}$, P. Bordas ${ }^{40}$, V. Bosch-Ramon ${ }^{40}$, S. Bottai ${ }^{25}$, P. Brogi ${ }^{36}$, F. Cadoux ${ }^{37}$, D. Campana ${ }^{31}$, W.W. Cao ${ }^{3}$, Z. Cao ${ }^{1}$, J. Casaus ${ }^{39}$, E. Catanzani ${ }^{35}$, P.W. Cattaneo ${ }^{33}$, J. Chang ${ }^{9}, 13$, Y.H. Chang ${ }^{21}$, G.M. Chen ${ }^{1}$, Y. Chen ${ }^{15}$, F. Cianetti ${ }^{35}$, A. Comerma ${ }^{40,41}$, D. Cortis ${ }^{28}$, X.H. Cui ${ }^{13}$, X.Z. Cui ${ }^{1}$, C. Dai ${ }^{5}$, Z.G. Dai ${ }^{15}$, R. D’Alessandro ${ }^{26}$, S. De Gaetano ${ }^{24}$, I. De Mitri2 ${ }^{27}$, F. de Palma ${ }^{30}$, V. Di Felice ${ }^{50}$, A. Di Giovanni ${ }^{27}$, M. Di Santo ${ }^{27}$, L. Di Venere ${ }^{24}$, J.N. Dong ${ }^{6,7}$, Y.W. Dong ${ }^{1}$, G. Donvito ${ }^{23}$, M. Duranti ${ }^{34}$, D. D’Urso ${ }^{49}$, C. Evoli ${ }^{27}$, K. Fang ${ }^{1}$, L. Fariña ${ }^{42}$, Y. Favre ${ }^{37}$, C.Q. Feng ${ }^{10}$, H. Feng ${ }^{16}$, H.B. Feng ${ }^{5}$, Z.K. Feng ${ }^{5}$, N. Finetti ${ }^{22}$, V. Formato ${ }^{50}$, J.M. Frieden ${ }^{44}$, P. Fusco ${ }^{24}$, J.R. Gao ${ }^{3}$, F. Gargano ${ }^{23}$, D. Gascon-Fora ${ }^{40}$, D. Gasparrini ${ }^{50}$, N. Giglietto ${ }^{24}$, F. Giovacchini ${ }^{39}$, S. Gomez ${ }^{40}$, K. Gong ${ }^{1}$, Q.B. Gou ${ }^{1}$, R. Guida ${ }^{46}$, D.Y. Guo ${ }^{1}$, J.H. Guo ${ }^{9}$, Y.Q. Guo ${ }^{1}$, H.H. He ${ }^{1}$, H.B. Hu ${ }^{1}$, J.Y. Hu ${ }^{1,2}$, P. Hu ${ }^{1,2}$, Y.M. Hu ${ }^{9}$, G.S. Huang ${ }^{10}$, J. Huang ${ }^{1}$, W.H. Huang ${ }^{6,7}$, X.T. Huang ${ }^{6,7}$, Y.B. Huang ${ }^{5}$, Y.F. Huang ${ }^{15}$, M. Ionica ${ }^{34}$, L. Jouvin ${ }^{42}$, A. Kotenko ${ }^{37}$, D. Kyratzis ${ }^{27}$, D. La Marra ${ }^{37}$,

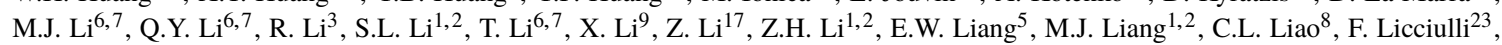

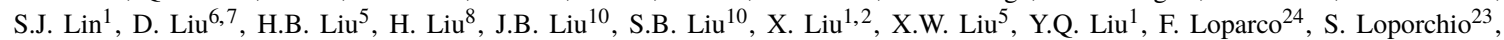
X. Lu ${ }^{5}$, J.G. Lyu ${ }^{4}$, L.W. Lyu ${ }^{3}$, P. Maestro ${ }^{36}$, E. Mancini ${ }^{34}$, R. Manera ${ }^{40}$, J. Marin ${ }^{39}$, P.S. Marrocchesi ${ }^{36}$, G. Marsella ${ }^{53}$, G. Martinez ${ }^{39}$, M. Martinez ${ }^{42}$, D. Marzullo ${ }^{47}$, J. Mauricio ${ }^{40}$, E. Mocchiutti ${ }^{45}$, G. Morettini $^{35}$, N. Mori ${ }^{25}$, L. Mussolin ${ }^{35}$, M.N. Mazziotta ${ }^{23}$, A. Oliva ${ }^{51}$, D. Orlandi ${ }^{28}$, G. Osteria ${ }^{31}$, L. Pacini ${ }^{25}$, B. Panico ${ }^{31}$, F.R. Pantaleo ${ }^{24}$, S. Papa ${ }^{46}$, P. Papini ${ }^{25}$, J.M. Paredes ${ }^{40}$, A. Parenti ${ }^{27}$, M. Pauluzzi ${ }^{35}$, M. Pearce ${ }^{43}$, W.X. Peng ${ }^{1}$, F. Perfetto ${ }^{31}$, C. Perrina ${ }^{44}$, G. Perrotta ${ }^{46}$, R. Pillera ${ }^{24}$, C. Pizzolotto ${ }^{45}$, R. Qiao ${ }^{1}$, J.J. Qin ${ }^{3}$, L. Quadrani ${ }^{51,52}$, Z. Quan ${ }^{1}$, A. Rappoldi ${ }^{33}$, G. Raselli ${ }^{33}$, X.X. Ren ${ }^{6,7}$, F. Renno ${ }^{46}$, M. Ribo ${ }^{40}$, J. Rico ${ }^{42}$, M. Rossella ${ }^{33}$, F. Ryde ${ }^{43}$, A. Sanmukh ${ }^{40}$, V. Scotti ${ }^{32}$, D. Serini ${ }^{23}$, D.L. Shi $^{3}$, Q.Q. Shi $^{6,7}$, L. Silveri ${ }^{27}$, O. Starodubtsev ${ }^{25}$, D.T. Su ${ }^{12}$, M. Su ${ }^{20}$, D. Sukhonos ${ }^{37}$, A. Suma ${ }^{46}$, X.L. Sun ${ }^{1}$, Z.T. Sun ${ }^{1,2}$, A. Surdo ${ }^{29}$, Z.C. Tang ${ }^{1}$, A. Tiberio ${ }^{26}$, A. Tykhonov ${ }^{37}$, V. Vagelli ${ }^{48}$, E. Vannuccini ${ }^{25}$, M. Velasco ${ }^{39}$, R. Walter $^{38}$, A.Q. Wang ${ }^{6,7}$, B. Wang ${ }^{3}$, J.C. Wang ${ }^{14}$, J.M. Wang ${ }^{1}$, J.J. Wang ${ }^{1,2}$, L. Wang ${ }^{13}$, M. Wang ${ }^{6,7}$, R.J. Wang ${ }^{1}$, S. Wang ${ }^{9}$, X.Y. Wang ${ }^{15}$, X.L. Wang ${ }^{10}$, Z.G. Wang ${ }^{1}$, D.M. Wei ${ }^{9}$, J.J. Wei ${ }^{9}$, B.B. Wu ${ }^{1}$, J. Wu ${ }^{19}$, L.B. Wu ${ }^{27}$, X. Wu ${ }^{37}$, X.F. Wu ${ }^{9}$, Y.L. Xin ${ }^{8},{\mathrm{M} . ~ X u^{1}}^{1}$, Z.Z. Xu ${ }^{10}$, H.R. Yan ${ }^{17}$, Y. Yang ${ }^{3}$, P.F. Yin ${ }^{1}$, Y.W. Yu ${ }^{18}$, Q. Yuan ${ }^{9}$, G. Zampa ${ }^{45}$, N. Zampa ${ }^{45}$, M. Zha ${ }^{1}$, C. Zhang ${ }^{1}$, F.Z. Zhang ${ }^{1,2}$, L. Zhang ${ }^{1}$, L. Zhang ${ }^{11}$, L.F. Zhang ${ }^{1,2}$, S.N. Zhang ${ }^{1,2}$, Y. Zhang ${ }^{9}$, Y.L. Zhang ${ }^{10}$, Z.G. Zhao ${ }^{10}$, J.K. Zheng ${ }^{3}$, Y.L. Zhou ${ }^{5}$, F.R. Zhu ${ }^{8}$, K.J. Zhu ${ }^{4}$

1 Key Laboratory of Particle and Astrophysics, Chinese Academy of Sciences, Beijing, China

2 University of Chinese Academy of Sciences, Beijing, China

Xi' an Institute of Optics and Precision Mechanics of CAS, Xi' an, China

Institute of High Energy Physics, Chinese Academy of Sciences, Beijing, China

School of Physical Science and Technology, Guangxi University, Nanning, China

Institute of Frontier and Interdisciplinary Science, Shandong University, Qingdao, China

Key Laboratory of Particle Physics and Particle Irradiation, Ministry of Education, China

8 School of Physical Science and Technology, Southwest Jiaotong University, Chengdu, China

9 Key Laboratory of Dark Matter and Space Astronomy, Purple Mountain Observatory, Chinese Academy of Sciences, Nanjing 210023, China

10 Department of Modern Physics, University of Science and Technology of China, Hefei, China

11 Department of Astronomy, Yunan University, Kunming, China

12 North Night Vision Technology co. Itd., Kunming, China

13 National Astronomical Observatories, Chinese Academy of Sciences, Beijing 100101, China

14 Yunnan Astronomical Observatory, Chinese Academy of Sciences, Kunming, China

15 School of Astronomy and Space Science, Nanjing University, Nanjing, China

16 Department of Astronomy, Tsinghua University, Beijing, China

17 Department of Astronomy, Peking University, Beijing, China

18 Institute of Astrophysics, Central China Normal University, Wuhan, China

19 Department of Physics, China University of Geosciences, Wuhan, China

20 The University of Hong Kong, Hong Kong, China

21 Institute of Physics, Academia Sinica, Taipei, Taiwan

22 Università dell'Aquila and Istituto Nazionale di Fisica Nucleare, Sezione di Firenze, Firenze, Italy

23 Istituto Nazionale di Fisica Nucleare, Sezione di Bari, Bari, Italy

24 Dipartimento di Fisica “M.Merlin” dell’Univerisità e del Politecnico di Bari, and Istituto Nazionale di Fisica Nucleare, Sezione di Bari, Bari, Italy

25 Istituto Nazionale di Fisica Nucleare, Sezione di Firenze, Firenze, Italy

26 Università di Firenze and Istituto Nazionale di Fisica Nucleare, Sezione di Firenze, Firenze, Italy

27 Gran Sasso Science Institute, L'Aquila, Italy and INFN Laboratori Nazionali del Gran Sasso, Assergi, L'Aquila, Italy

28 INFN Laboratori Nazionali del Gran Sasso, L'Aquila, Italy

29 Istituto Nazionale di Fisica Nucleare, Sezione di Lecce, Lecce, Italy

30 Universitá del Salento and Istituto Nazionale di Fisica Nucleare, Sezione di Lecce, Lecce, Italy

31 Istituto Nazionale di Fisica Nucleare, Sezione di Napoli, Napoli, Italy

32 Università di Napoli "Federico II" and Istituto Nazionale di Fisica Nucleare, Sezione di Napoli, Napoli, Italy

33 Istituto Nazionale di Fisica Nucleare, Sezione di Pavia, Pavia, Italy 
34 Istituto Nazionale di Fisica Nucleare, Sezione di Perugia, Perugia, Italy

35 Università degli Studi di Perugia and Istituto Nazionale di Fisica Nucleare, Sezione di Perugia, Perugia, Italy

36 Università di Siena and Istituto Nazionale di Fisica Nucleare, Sezione di Pisa, Pisa, Italy

37 Département de Physique Nucléaire et Corpusculaire (DPNC), Université de Genève, Genève, Switzerland

38 Department of Astronomy, University of Geneva, Geneva, Switzerland

39 Centro de Investigaciones Energéticas, Medioambientales y Tecnológicas (CIEMAT), E-28040 Madrid, Spain

40 Dept. Física Quàntica i Astrofísica, Institut de Ciències del Cosmos (ICCUB), Universitat de Barcelona (IEEC-UB), Barcelona, Spain

41 Universitat Pompeu Fabra (UPF), Barcelona, Spain

42 Institut de Física d'Altes Energies (IFAE), The Barcelona Institute of Science and Technology (BIST), E-08193 Bellaterra, Barcelona, Spain

43 KTH Royal Institute of Technology, Stockholm, Sweden

44 Institute of Physics, Ecole Polytechnique Fédérale de Lausanne (EPFL), Lausanne, Switzerland

45 Istituto Nazionale di Fisica Nucleare, Sezione di Trieste, Trieste, Italy

46 Università degli Studi di Napoli Federico II, Napoli, Italy

47 Università degli Studi di Trieste, Italy

48 Italian Space Agency and Istituto Nazionale di Fisica Nucleare, Sezione di Perugia, Perugia, Italy

49 Università degli Studi di Sassari and Istituto Nazionale di Fisica Nucleare, Sezione di Perugia, Perugia, Italy

50 Istituto Nazionale di Fisica Nucleare, Sezione di Roma Tor Vergata, Rome, Italy

51 Istituto Nazionale di Fisica Nucleare, Sezione di Bologna, Bologna, Italy

52 Università di Bologna and Istituto Nazionale di Fisica Nucleare, Sezione di Bologna, Bologna, Italy

53 Dipartimento di Fisica e Chimica "E. Segrè" Università degli Studi di Palermo, Palermo, Italy and INFN sez. Catania, Catania, Italy 